See discussions, stats, and author profiles for this publication at: https://www.researchgate.net/publication/348606373

Rhombohedral distortion-driven ferroelectric order and exchange bias effect in geometrically frustrated $\mathrm{ZnFe} 2 \mathrm{O} 4$

Article in Physical Review Materials · January 2021

Dol: 10.1103/PhysRevMaterials.5.014410

1 author:

Jayjit Kumar Dey

Indian Association for the Cultivation of Science

6 PUBLICATIONS 36 CITATIONS

SEE PROFILE 


\title{
Rhombohedral distortion-driven ferroelectric order and exchange bias effect in geometrically frustrated $\mathrm{ZnFe}_{2} \mathrm{O}_{4}$
}

\author{
J. K. Dey, ${ }^{1}$ A. Chatterjee, ${ }^{1}$ A.-C. Dippel, ${ }^{2}$ O. Gutowski, ${ }^{2}$ M. v. Zimmermann, ${ }^{2}$ S. Majumdar, ${ }^{1}$ and S. Giri ${ }^{1, *}$ \\ ${ }^{1}$ School of Physical Sciences, Indian Association for the Cultivation of Science, Jadavpur, Kolkata 700032, India \\ ${ }^{2}$ Deutsches Elektronen-Synchrotron DESY, Notkestrasse 85, 22607 Hamburg, Germany
}

(Received 27 July 2020; accepted 5 January 2021; published 19 January 2021)

\begin{abstract}
The rhombohedral distortion-driven occurrence of the spontaneous electric polarization at a reasonably high temperature $(T)$ and an exchange bias (EB) effect below the antiferromagnetic (AFM) Néel temperature $\left(T_{N}\right)$ are revealed in $\mathrm{ZnFe}_{2} \mathrm{O}_{4}$. We observe the magnetic memory effect, suggesting a cooperative glassy magnetic state below $T_{N}$. The phase separation between the long-range AFM and the glassy magnetic component leads to the EB effect below $T_{N}$. The synchrotron diffraction studies confirm a structural transition to a polar $R 3 \mathrm{~m}$ structure from the cubic spinel structure, involving a strong rhombohedral distortion. This distortion correlates with the occurrence of the spontaneous electric polarization $(P)$ below $\sim 110 \mathrm{~K}\left(T_{\mathrm{FE} 1}\right)$, which is accompanied by a short-range order (SRO). The $P$-value enhances further due to an additional rhombohedral distortion below $T_{N}$. A considerable magnetoelectric (ME) coupling is detected below $T_{\mathrm{FE} 1}$. The increase of $P$ is $\sim 7.2 \%$ for $5 \mathrm{~T}$ at the liquid nitrogen temperature. There has been a fundamental interest among the community in the unique result of the occurrence of ferroelectric (FE) order coexisting with SRO and having a significant ME coupling, which is accompanied by a strong rhombohedral structural distortion analogous to that observed in $\mathrm{BiFeO}_{3}$.
\end{abstract}

DOI: 10.1103/PhysRevMaterials.5.014410

\section{INTRODUCTION}

$A B_{2} X_{4}$-type compounds continue to inspire interest in magnetism due to the tuning of the $A$ and $B$ atoms, which are crystallized in the spinel structure. Here, $X$ represents either the oxygen or the chalcogenides. For example, the solely $A$ site magnetic atom in the spinel structure gives rise to exotic effects such as spin dimerization [1], spin liquid ground states [2-4], and suppressed antiferromagnetic order [5], which are often regulated by either orbital ordering or geometric magnetic frustrations, or both. Transition elements in both the $A$ and $B$-sites reduce the magnetic frustration, but this produces interesting consequences such as structural instabilities [6-8], intricate magnetic structures [9,10], ferroelectricity [11-14], etc. The solely $B$-site magnetic atom in $A B_{2} X_{4}$ is found to have potential for the pyrochlore structure, producing strong geometric magnetic frustration $[15,16]$. The geometrically frustrated pyrochlore structure becomes the center of attraction when it reveals interesting fallout, specifically various spin ice states [17-19], charge ordering [20,21], and intricate magnetic structures [22,23].

The spinel $\mathrm{Zn}$-ferrite $\mathrm{ZnFe}_{2} \mathrm{O}_{4}(\mathrm{ZFO})$ attracts special attention due to the $B$-site magnetic frustration, which is attributed to the pyrochlore structure [24]. An example of a pyrochlore structure formed solely by $\mathrm{Fe}$ atoms in the $F d \overline{3} m$ structure is depicted in Fig. 1(a). The structure is composed of corner-sharing tetrahedra, where four corners of a tetrahedron are occupied by the $\mathrm{Fe}$ atom. In the case of antiferromagnetic (AFM) exchange interaction, a tetrahedral unit is highly frustrated, as depicted by the question marks in Fig. 1(b).

*sspsg2@iacs.res.in
Long-range AFM order at $T_{N}$ has been reported, suggesting $T_{N}$ is in the range of $10.5-13 \mathrm{~K}$ by means of the neutron, $\mu$-SR, and Mössbauer studies [25,26]. The $T_{N}$ at $13 \mathrm{~K}$ was proposed with a high paramagnetic Curie-Weiss temperature, $\Theta=120 \mathrm{~K}$, which provided a high value of the $\Theta / T_{N}$ ratio, pointing to strong magnetic frustration [26]. The density functional theory further interpreted the nature of the AFM order $[27,28]$. Revisiting the Mössbauer studies, it was proposed that the disorder, either introduced by the external pressure or the internal pressure attributed to the chemical disorder, was found to be crucial for tuning the magnetism of ZFO [29-32]. The external pressure effect demonstrated an irreversible change in the unit-cell volume, correlating with a change in the spin state [31]. The chemical disorder-driven spin-glass and/or cluster-glass state was proposed [32], which was also interpreted from $a b$ initio calculations [33]. The magnetic diffuse scattering results proposed a spin liquid state, analogous to the three-dimensional (3D) quantum spin liquid system, $\mathrm{Y}(\mathrm{Sc}) \mathrm{Mn}_{2}$, which was attributed to the strong magnetic frustration [34,35]. For the dominant short-range order (SRO), preferably, the existence of a ferromagnetic (FM) cluster has been proposed in nanoscale ZFO from neutron polarization studies [36]. In fact, a dominant SRO has been proposed from the neutron diffraction studies of a ZFO crystal, which was suggested to occur around 10 times $(\sim 105 \mathrm{~K})$ for $T_{N}$ at $10.5 \mathrm{~K}$, and also proposed to exist a SRO state even below $T_{N}$ [25]. Up to now, a lot of attention has been paid to the magnetic ground state below $T_{N}$ using different microscopic experimental tools, although the physical origin of the $\mathrm{SRO}$, observed at much higher than $T_{N}$, remains unaddressed.

In the article, we report significant magnetoelastic coupling close to the proposed emergence of SRO for ZFO. We observe a structural transition to a polar structure with a $R 3 m$ 
(a)

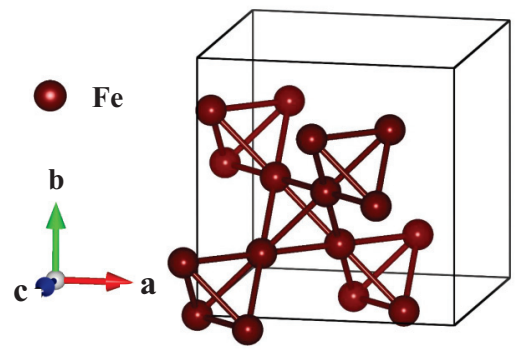

(b)

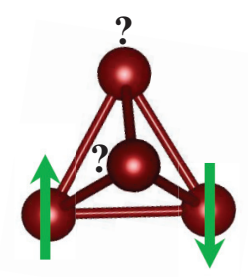

FIG. 1. (a) A part of the pyrochlore structure formed by the Fe atoms crystallized in $F d \overline{3} m$ structure within the unit cell. (b) Antiferromagnetically coupled tetrahedron unit, with examples of the magnetic frustrations denoted by the "?" marks.

space group from the cubic $F d \overline{3} m$ structure around $\sim 110 \mathrm{~K}$. Intriguingly, this structural transition is associated with a ferroelectric (FE) order with the ordering at $T_{\mathrm{FE} 1}=110 \mathrm{~K}$, which is followed by an evident signature of magnetoelectric (ME) coupling at $T_{N}$. The value of electric polarization $(P)$ is considerable as $\sim 125 \mu \mathrm{C} / \mathrm{m}^{2}$ at liquid nitrogen temperature for a $500 \mathrm{kV} / \mathrm{m}$ poling field. The value of $P$ increases with the application of a magnetic field $(B)$, pointing to ME coupling. The $P$ increases up to $\sim 7.2 \%$ for $B=5 \mathrm{~T}$ at liquid nitrogen temperature. The dc magnetization results show the magnetic memory effect in the thermal variation as well as relaxation dynamics below $T_{N}$, pointing to a signature of a cooperative glassy magnetic behavior. The glassy magnetic component coexisting with the AFM ordered state leads to the emergence of an exchange bias (EB) effect due to the field-cooling below $T_{N}$. The value of the EB field is reasonable: $\sim 6.7 \mathrm{mT}$ at $2 \mathrm{~K}$ for a cooling field of $1 \mathrm{~T}$. Current investigation has unfolded the important issues close to $T_{N}$ and $T_{\mathrm{FE} 1}$. At $T_{N}$, a strong rhombohedral distortion is proposed, which is accompanied by an increase in the FE polarization and a magnetic phase separation, giving rise to the EB effect. The structural transition to a polar $R 3 m$ structure at $T_{\mathrm{FE} 1}$, which involves a strong rhombohedral distortion, is very similar to the observed ferroelectricity driven by the rhombohedral distortion in the $R 3 m$ structure for $\mathrm{BiFeO}_{3}[37,38]$.

\section{EXPERIMENTAL RESULTS}

Polycrystalline $\mathrm{ZnFe}_{2} \mathrm{O}_{4}$ is prepared using a standard solid-state reaction technique [25]. The chemical composition is checked by $\mathrm{x}$-ray diffraction at room temperature recorded in a PANalytical x-ray diffractometer (model: $X$ ' Pert PRO) using $\mathrm{Cu} K \alpha$ radiation. The single-phase chemical composition is further confirmed by the synchrotron diffraction recorded with a wavelength of $0.1422 \AA(87.1 \mathrm{keV})$ at the P07 beamline of PETRA III, Hamburg, Germany. The synchrotron diffraction data are analyzed using Rietveld refinement with commercially available MAUD software. The polycrystalline sample, pressed into a pellet, is used for the dielectric measurements, using an E4980A LCR meter (Agilent Technologies, USA) equipped with a commercial PPMS Evercool-II system of Quantum Design. The pyroelectric current $\left(I_{p}\right)$ is recorded in an electrometer (Keithley, model 6517B) at a constant temperature sweep rate. In all

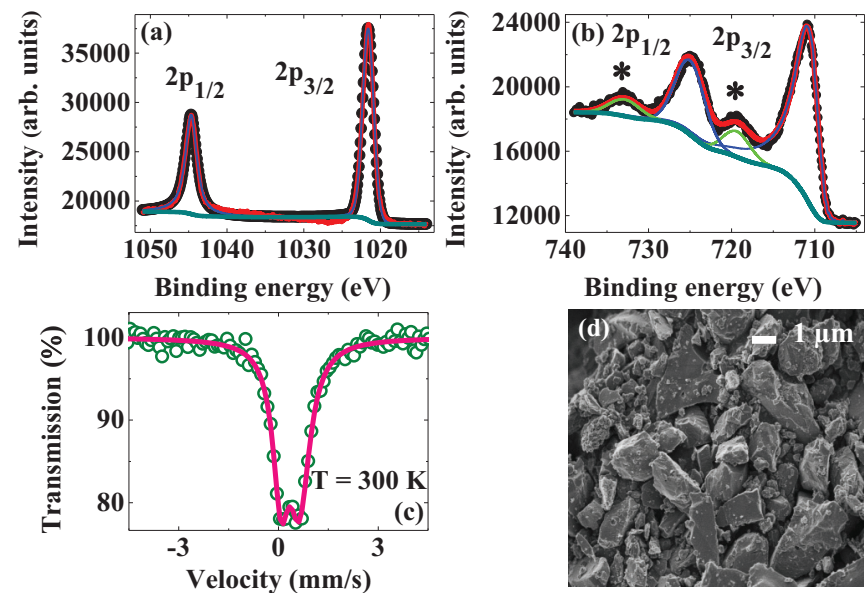

FIG. 2. (a) Zn $2 p$ and (b) Fe $2 p$ core level XPS of ZFO. (c) ${ }^{57} \mathrm{Fe}$ Mössbauer spectrum recorded in the transmission mode at $300 \mathrm{~K}$. The fits in (a), (b), and (c) are shown by the solid curves. (d) SEM image highlighting the particles.

the measurements, the electrical contacts are fabricated using air-drying silver paint. The poling electric fields are applied during cooling processes, and the values of $I_{p}$ are measured in the warming mode in zero electric field. Before the measurement of $I_{p}$, the electrical connections are short-circuited and left to wait for a sufficiently long time. Magnetization is measured in a commercial magnetometer of Quantum Design (MPMS, Evercool). Scanning electron microscopy (SEM) is performed using a JEOL JSM-6010LA. X-ray photoemission spectroscopy (XPS) is recorded with a spectrometer of Omicron Nanotechnology. The Mössbauer spectrum is recorded in a transmission geometry using a ${ }^{57} \mathrm{Co}$ source fixed in a $\mathrm{Rh}$ matrix, where a Wissel velocity drive unit is used in a constant acceleration mode. The Mössbauer parameters are estimated with respect to the $\alpha-\mathrm{Fe}$.

\section{EXPERIMENTAL RESULTS AND DISCUSSIONS}

\section{A. Chemical and physical characterization}

The $\mathrm{Zn} 2 p$ and Fe $2 p$ core level XPS of ZFO are summarized in Figs. 2(a) and 2(b). In Fig. 2(a), the two peaks observed at 1021.6 and $1044.7 \mathrm{eV}$ correspond to the $2 p_{3 / 2}$ and $2 p_{1 / 2}$ peaks of $\mathrm{Zn}$, respectively. Figure $2(\mathrm{~b})$ depicts the spectrum with the peaks at 725 and $710.9 \mathrm{eV}$ for $\mathrm{Fe} 2 p_{1 / 2}$ and $2 p_{3 / 2}$, respectively. The satisfactory fits are shown by the solid curves on the experimental data in both figures. The results indicate the $\mathrm{Zn}^{2+}$ and $\mathrm{Fe}^{3+}$ states in $\mathrm{ZFO}$, as reported earlier [39-41]. We note that the ratio of the area under the curve of the $\mathrm{Zn}$ - and $\mathrm{Fe}$-spectrum is close to $1: 2$, which is reasonable for the composition of $\mathrm{ZnFe}_{2} \mathrm{O}_{4}$. To further confirm the oxidation state of Fe, the Mössbauer spectrum is recorded at $300 \mathrm{~K}$, as depicted in Fig. 2(c). The paramagnetic state, as indicated by the doublet spectrum, is realized with an isomer shift (IS) of $0.36 \mathrm{~mm} / \mathrm{s}$ and a quadrupole splitting (QS) of 0.45 $\mathrm{mm} / \mathrm{s}$. The value of the IS is consistent with that observed for the high-spin $\mathrm{Fe}^{3+}$ state [29,30]. Figure 2(d) illustrates the SEM image. The average grain size is obtained using the mean lineal intercept technique, $D_{\text {average }}=1.56 \mathrm{~L} / \mathrm{MN}$, where 

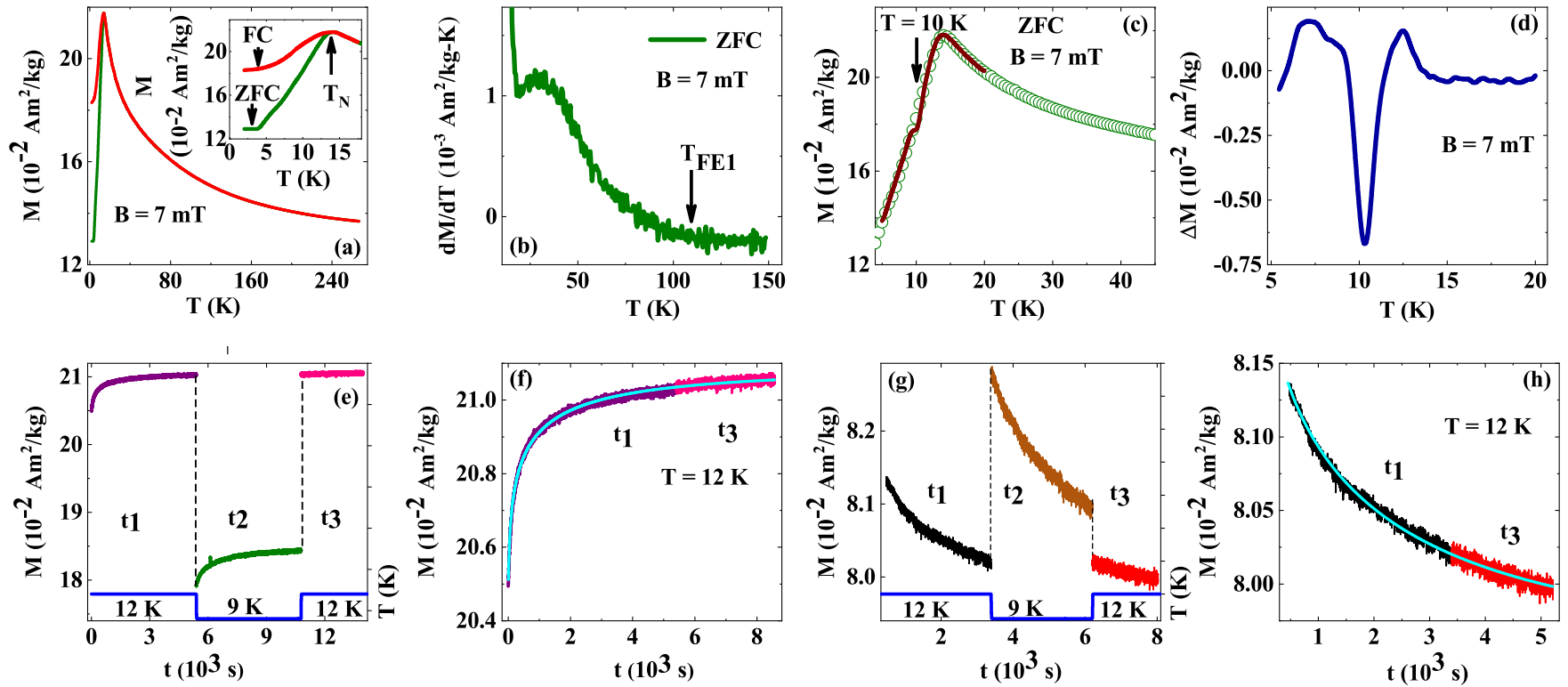

FIG. 3. (a) The $T$ variations of ZFC-FC magnetization curves recorded at $7 \mathrm{mT}$. The arrow in the inset highlights $T_{N}$, below which ZFC and FC magnetization curves deviate from each other. (b) The $d M / d T$ with $T$ recorded in $\mathrm{ZFC}$ mode, highlighting $T_{\mathrm{FE} 1}$. (c) The $T$ variation of the ZFC curve recorded at $7 \mathrm{mT}$, defined as a reference curve and the memory curve, as indicated by the solid curve. The arrow indicates the aging temperature at $10 \mathrm{~K}$. (d) Difference between these curves shown by $\Delta M$ plotted with $T$, highlighting a sharp "dip" close to $10 \mathrm{~K}$. (e) The $t$ dependence of $M$ at $12 \mathrm{~K}, 9 \mathrm{~K}$, and again at $12 \mathrm{~K}$ for $t 1, t 2$, and $t 3$, respectively, with $7 \mathrm{mT}$ after cooling in ZFC mode. (f) The $t$ dependence of $M$ at $12 \mathrm{~K}$ for $t 1+t 3$ recorded at $7 \mathrm{mT}$, following a single stretched exponential function. (g) The $t$ dependence of $M$ at $12 \mathrm{~K}$, $9 \mathrm{~K}$, and again at $12 \mathrm{~K}$ for $t 1, t 2$, and $t 3$, respectively in zero-field after cooling in FC mode with a field of $7 \mathrm{mT}$. (f) $t$ dependence of $M$ at $12 \mathrm{~K}$ for $t 1+t 3$ in zero-field, following a single stretched exponential function. The $T$ vs $t$ plots for the experiments in ZFC and FC modes are shown at the bottom of panels (e) and (g), respectively.

$D_{\text {average }}$ is the average grain size, $L$ is the total length of test line used, $N$ is the number of intercepts, and $M$ is the magnification of the SEM image. Here, the proportionality constant, 1.56 , is essentially a correction factor, which was derived by Mendelson [42]. The value of $D_{\text {average }}$ is $2.6 \mu \mathrm{m}$ with a standard deviation of 0.33 .

\section{B. Short-range order above $T_{N}$}

Thermal variations of magnetization $(M)$ are recorded in the zero-field-cooled (ZFC) and field-cooled (FC) protocols, which are shown in Fig. 3(a) for $B=7 \mathrm{mT}$. A sharp peak is observed in both the ZFC and FC curves, below which they deviate from each other at $13.5 \mathrm{~K}\left(T_{N}\right)$, as depicted in the inset of the figure. The result is consistent with the reported results [26]. Figure 3(b) depicts a derivative plot of $M(d M / d T)$ recorded in the ZFC mode with temperature $(T)$. The curve indicates that with decreasing temperature it starts to increase slowly below $T_{\mathrm{FE} 1}$, as indicated by an arrow. Here, $T_{\mathrm{FE} 1}$ denotes the FE Curie temperature, which is described elsewhere in the text. The $d M / d T(T)$ curve is consistent with the previous report, indicating the occurrence of SRO [25]. Here, we note that the SRO starts to appear significantly close to $T_{\mathrm{FE} 1}$.

\section{Magnetic memory effect below $\boldsymbol{T}_{N}$}

To investigate the magnetic memory effect, the sample is cooled from $300 \mathrm{~K}$ down to $10 \mathrm{~K}\left(<T_{N}\right)$ in zero field and left to wait for $3600 \mathrm{~s}\left(t_{w}\right)$, which is followed by further cooling down to $2 \mathrm{~K}$. Next, the magnetization is recorded in the warming mode with $7 \mathrm{mT}$ for a temperature sweep rate of $1 \mathrm{~K} / \mathrm{min}$. The magnetization curve is recorded up to $20 \mathrm{~K}$, which is depicted by a continuous curve with $T$ in Fig. 3(c). We notice an apparent signature of anomaly at $10 \mathrm{~K}$, which is further compared with the reference curve, as shown by the open symbols in the figure. Here, the reference curve is the same ZFC curve as shown in Fig. 3(a). The difference between these two, defined as $\Delta M$, is depicted in Fig. 3(d). The $\Delta M$ versus $T$ plot clearly shows a "dip" at $10 \mathrm{~K}$, revealing the memory effect. The result is consistent with that observed for spin-glass systems [43]. During the aging process at $10 \mathrm{~K}$, the system is allowed to relax and it rearranges the spin configuration toward the equilibrium. This equilibrium state is frozen with a further cooling process, which can be retrieved during measurement on reheating the system. The magnetic memory effect observed in the ZFC mode suggests the cooperative glassy dynamics, as commonly observed for the spin glasses [43].

To verify the memory effect further, experiments on the relaxation dynamics are carried out using the experimental protocols proposed in the literature [44]. All the experimental results are summarized in Figs. 3(e)-3(h). At first, the relaxation of $M$ is recorded for a time period of $t 1$ at $12 \mathrm{~K}$ (below $T_{N}$ ) with $7 \mathrm{mT}$, after cooling the sample in zero-field from $300 \mathrm{~K}$. At the end of $t 1$, the sample temperature is lowered to $9 \mathrm{~K}$ and the relaxation is recorded for $t 2$. Finally, the sample temperature is heated back to $12 \mathrm{~K}$ and the relaxation is recorded for $t 3$, as shown in Fig. 3(e). The changes of temperature with time are depicted at the bottom of the figure. 
As displayed in Fig. 3(f), the relaxation processes during $t 1+t 3$ at $12 \mathrm{~K}$ follow a single stretched exponential function $[45,46]$. Similar results are observed for the relaxation dynamics measured in zero field at $12 \mathrm{~K}$ after cooling the sample in FC mode with $B=7 \mathrm{mT}$ from $300 \mathrm{~K}$, as shown in Fig. $3(\mathrm{~g})$. Similar to the experiment in the ZFC mode, the changes in temperature with time during the experiment are depicted at the bottom of the figure. Figure 3(h) depicts the relaxation process in zero field for $t 1+t 3$ at $12 \mathrm{~K}$, following a single stretched exponential function. The results demonstrate that the relaxation process after the temporary cooling for $T-\Delta T$ retrieves the previous history, pointing to the manifestations of the memory effect in the relaxation dynamics. Thus, the memory effects in both the thermal variation as well as relaxation dynamics suggest a glassy magnetic state. The glassy magnetic state, coexisting with the long-range AFM order below $T_{N}$, is analogous to that observed for $\mathrm{ZnCr}_{2} \mathrm{O}_{4}$ [47]. A considerable EB effect was observed for $\mathrm{ZnCr}_{2} \mathrm{O}_{4}$, which was attributed to the coexisting magnetic phases below $T_{N}$ [47].

\section{Exchange bias effect below $\boldsymbol{T}_{N}$}

Figure 4(a) depicts the magnetic hysteresis loop recorded at $2 \mathrm{~K}$, having a considerable coercive field $\left(H_{C}\right)$. As depicted in the inset of the figure, the loop closes around $\sim 1.4 \mathrm{~T}$, above which almost a linear magnetization curve is observed, as noted for the AFM ground state. The opening of the loop in the low-field region for an AFM ground state indicates an additional magnetic phase coexisting with the AFM phase below $T_{N}$, which has also been reported earlier from the combined neutron, $\mu$-SR, and Mössbauer studies [25]. Here, the glassy magnetic component, as indicated by the memory effect, coexisting with the AFM component, may lead to the occurrence of the EB effect even in a chemically single phase compound [48]. To test it, initially, we cool the sample down to $2 \mathrm{~K}$ with the cooling field of $\pm 1 \mathrm{~T}$ and the hysteresis loop is measured in between $\pm 5 \mathrm{~T}\left(H_{\max }\right)$, as depicted in Fig. 4(a). The inset of the figure highlights that the loop closes around $\sim 1.4 \mathrm{~T}$, which is less than $1 / 3$ of $H_{\max }$. A negative shift for the positive cooling field and a positive shift for the negative cooling field are depicted in Figs. 4(b) and 4(c), respectively. This manifestation is typical for the EB effect [49-53]. We further note that the field-cooling from the temperatures above $T_{\mathrm{FE} 1}$ or $T_{N}$ does not significantly influence the EB effect. From the shift of the hysteresis loop in the field axis, the EB field is defined as $H_{E}=-\left(H_{1}+H_{2}\right) / 2$ and $H_{C}=\left(H_{2}-H_{1}\right) / 2$ for a positive cooling field, where $H_{1}$ and $H_{2}$ are the left and right coercive fields, respectively.

The values of $H_{E}$ and $H_{C}$ at $2 \mathrm{~K}$ for a cooling field of $1 \mathrm{~T}$ are depicted in Figs. 4(d) and 4(e), respectively. Initially, the EB field increases with increasing cooling field up to $1 \mathrm{~T}$, which is associated with the increase in the coercive field. This observation is an indication of the appearance of a magnetic phase with the considerably increased anisotropy driven by the cooling field. Thus the field-cooling process gives rise to the emergence of a new layer composed of the pinned spins at the interface between the AFM and the glassy magnetic phases. The possible occurrence of a pinned layer at the interface between the AFM and glassy magnetic layer is illustrated in the inset of Fig. 4(d). The pinned layer, carrying
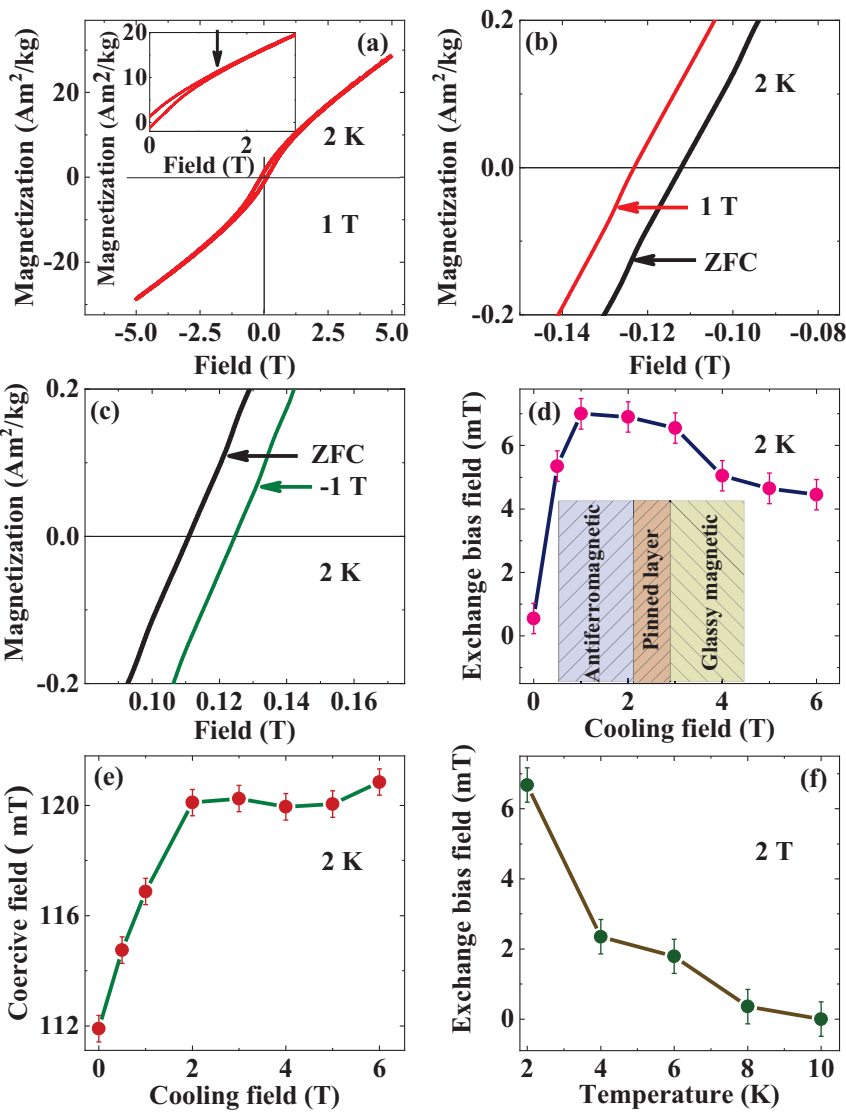

FIG. 4. (a) Hysteresis loop recorded up to maximum field of $\pm 5 \mathrm{~T}$ at $2 \mathrm{~K}$ for a cooling field of $1 \mathrm{~T}$. The inset highlights that the loop closes around 1.4 T, as indicated by an arrow. (b) Negative and (c) positive shifts are shown for a cooling field of $\pm 1 \mathrm{~T}$. Cooling field dependence of (d) EB field and (e) coercive field at $2 \mathrm{~K}$. Temperature variation of (f) EB field for a cooling field of $2 \mathrm{~T}$. The inset of (d) illustrates a representative illustration of the occurrence of a pinned layer at the interface between AFM and glassy magnetic phases.

the unidirectional anisotropy, causes a systematic shift of the hysteresis loop driven by the cooling field and leads to the EB effect. This unidirectional anisotropy strongly depends on the individual anisotropy of the magnetic phases coupled with the pinned layer. We note that with a further increase in the cooling field above $1 \mathrm{~T}$, the EB field shows a decreasing trend, which is often observed for the exchange bias effect, involving the glassy magnetic component [54-57]. When the cooling field is high, the frozen spins, belonging to the glassy magnetic component, are almost fully aligned and vary a little along the field with a further increase in the higher cooling field. As a result of it, the magnetic coupling between the strong cooling field and the glassy magnetic moment increases, tending to polarize them along the field direction, where the magnetic coupling dominates over the EB coupling. This may lead to a decrease in the EB field at the higher cooling field. As depicted in Fig. 4(f), the EB field decreases with decreasing temperature and nearly vanishes close to $T_{N}$. The result indicates that the AFM state below $T_{N}$ leads to a crucial role for the EB effect. 

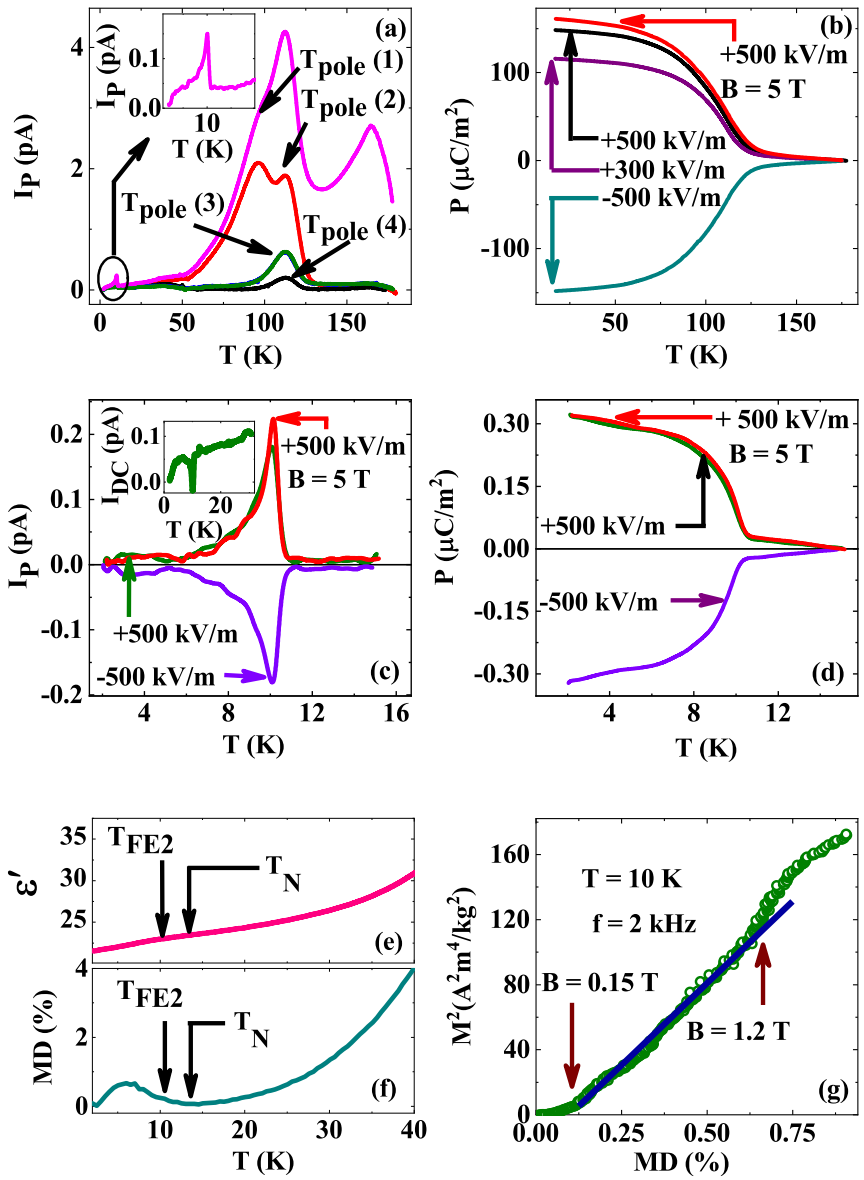

FIG. 5. The $T$ variations of (a) $I_{p}$ recorded with a constant $T$ sweep rate of $5 \mathrm{~K} / \mathrm{min}$ at different poling temperatures $\left(T_{\text {pole }}\right)$, as described in the text and for a poling field $(E)$ of $500 \mathrm{kV} / \mathrm{m}$; (b) $P$ for $E=+300, \pm 500 \mathrm{kV} / \mathrm{m}$, and $500 \mathrm{kV} / \mathrm{m}$ with a magnetic field of $5 \mathrm{~T}$; (c) $I_{p}$ for $E= \pm 500 \mathrm{kV} / \mathrm{m}$ and the same with a magnetic field of $5 \mathrm{~T}$ for $T_{\text {pole }}$ at $15 \mathrm{~K}$; and (d) $P$, as calculated from the data given in (c). The inset of (a) highlights another low- $T$ peak and (c) shows the dc bias current $\left(I_{\mathrm{DC}}\right)$ with $T$ with a "dip" at $\sim 10.5 \mathrm{~K}$. The $T$ variations of (e) $\epsilon^{\prime}$ at $f=2 \mathrm{kHz}$ and (f) $\mathrm{MD}(\%)$, showing $T_{\mathrm{FE} 2}$ and $T_{N}$ by the arrows. Plot of (g) $M^{2}$ with $\mathrm{MD}(\%)$ at $10 \mathrm{~K}$ for $f=2 \mathrm{kHz}$.

\section{E. Ferroelectric order above $\boldsymbol{T}_{N}$}

Figure 5(a) shows a temperature variation of the pyroelectric current $\left(I_{p}\right)$ recorded with the poling electric field of 500 $\mathrm{kV} / \mathrm{m}$ and a thermal sweep rate of $5 \mathrm{~K} / \mathrm{min}$ in the different poling conditions. To confirm the peak in $I_{p}(T)$, appearing due to a genuine occurrence of the polar order, the values of $I_{p}(T)$ are recorded for the different poling temperatures. In the figure, $T_{\text {pole }}(1), T_{\text {pole }}(2), T_{\text {pole }}(3)$, and $T_{\text {pole }}(4)$ represent the poling temperatures at $150,95,20$, and $8 \mathrm{~K}$, respectively. In all the cases, a peak at $110 \mathrm{~K}$ is always observed, pointing to a genuine occurrence of the peak $[7,58,59]$, which is shown in the figure. For $T_{\text {pole }}(1)$ another high-temperature peak is observed, which disappears with $T_{\text {pole }}$ close to or below the polar order. The high- $T$ peak appears due to the extrinsic thermally stimulated depolarization currents (TSDC) [7,58-60]. For the poling at $95 \mathrm{~K}$, an additional peak close to $95 \mathrm{~K}$ appears below the peak at $110 \mathrm{~K}$. The peak around $95 \mathrm{~K}$ disappears for the poling at 150,20 , and $8 \mathrm{~K}$, although the peak at $110 \mathrm{~K}$ is always evident, which is described as $T_{\mathrm{FE} 1}$. After subtracting the TSDC component for $T_{\text {pole }}$ at $150 \mathrm{~K}$, the $I_{p}(T)$, integrated with time, provides the thermal variation of $P$. The $P(T)$ curves are depicted in Fig. 5(b) for different poling fields. The $P(T)$ curve reverses almost symmetrically due to a change of sign of the poling field, pointing to the $\mathrm{FE}$ nature of the polar order. The value of $P$ at $15 \mathrm{~K}$ is $\sim 148 \mu \mathrm{C} / \mathrm{m}^{2}$ for a poling field of $500 \mathrm{kV} / \mathrm{m}$. With the application of a magnetic field, the $P$-value increases, pointing to a significant ME. The changes of $P$ due to a 5 T magnetic field are $7.2(\%)$ and $8.6(\%)$ at the liquid nitrogen temperature and $17 \mathrm{~K}$, respectively.

We note that another peak is observed in $I_{p}(T)$ close to $T_{N}$ for $T_{\text {pole }}$ (1), as highlighted in the inset of Fig. 5(a). To focus on this low- $T$ peak, the poling is further done at $15 \mathrm{~K}$ with a temperature sweep rate of $5 \mathrm{~K} / \mathrm{min}$ for a poling field of $\pm 500 \mathrm{kV} / \mathrm{m}$. As depicted in Fig. 5(c), the $I_{p}(T)$ exhibits a sharp peak at $10.5 \mathrm{~K}$, which reverses due to a change in sign of the poling field, pointing to the FE nature of the additional polar order below $T_{N}$. The inset of the figure shows a sharp "dip" in the bias current $\left(I_{\mathrm{DC}}\right)$, which is recorded in the heating cycle with a thermal sweep rate of $5 \mathrm{~K} / \mathrm{min}$ and a bias electric field of $500 \mathrm{kV} / \mathrm{m}$. Thus the sharp peak in $I_{p}(T)$ is genuinely observed at $10.5 \mathrm{~K}\left(T_{\mathrm{FE} 2}\right)$. Integration of $I_{p}(T)$ with time provides $P(T)$, which is shown in Fig. 5(d). With the application of a $5 \mathrm{~T}$ magnetic field, the $P(T)$ does not change convincingly, as also indicated in Fig. 5(d).

\section{F. Dielectric and magnetodielectric effect}

Dielectric permittivity $(\epsilon)$ is recorded with $T$ at a fixed frequency $(f)$, for example at $f=2 \mathrm{kHz}$ for a magnetic field $(B)$ of 0 and 5 T. Thermal variation of the real $\left(\epsilon^{\prime}\right)$ component of $\epsilon$ is shown in Fig. 5(e). We note that the value of $\epsilon^{\prime}$ is not reliable above $\sim 40 \mathrm{~K}$, which is attributed to the low resistivity. To test the reliable value of $\epsilon^{\prime}$ below $\sim 40 \mathrm{~K}$, we calculate the value of $P / E$, which is found close to the value of $\epsilon^{\prime}$. The value of $P / E$ is $\sim 33$ for $E=500 \mathrm{kV} / \mathrm{m}$, which is quite reasonable for $\epsilon^{\prime} \approx 24$ at $16 \mathrm{~K}$. This discrepancy may be attributed to the nonlinear behavior of the $P-E$ curve close to $E=500 \mathrm{kV} / \mathrm{m}$. The percentage of magnetodielectric (MD) response, defined as $\left[\epsilon^{\prime}(B) / \epsilon^{\prime}(B=0)-1\right] \times 100$, is depicted with $T$ for $B=5 \mathrm{~T}$ in Fig. 5(f). $T_{N}$ and $T_{\mathrm{FE} 2}$ are highlighted by the arrows. A minimum in $\operatorname{MD}(T)$ is noted around $T_{N}$. Below $T_{\mathrm{FE} 2}$, the $\mathrm{MD}(T)$ shows a maximum around $7 \mathrm{~K}$.

The MD consequences are correlated to the ME coupling, which have been phenomenologically expressed using the Ginzburg-Landau theory through the ME coupling term $\gamma P^{2} M^{2}$ in the thermodynamic potential $(\Phi)$, which is defined as

$$
\begin{aligned}
\Phi= & \Phi_{0}+\alpha P^{2}+\frac{\beta}{2} P^{4}-P E+\alpha^{\prime} M^{2}+\frac{\beta^{\prime}}{2} M^{4}-M B \\
& +\gamma P^{2} M^{2} .
\end{aligned}
$$

Here, $\alpha, \beta, \alpha^{\prime}, \beta^{\prime}$, and $\gamma$ are the constants and functions of temperature. In the magnetically ordered state, the influence of magnetic order on the MD response is followed by a linear response of the $M^{2}$ versus $\mathrm{MD}(\%)$ curve. Here, the $M^{2}$ with $\mathrm{MD}(\%)$ plot at $10 \mathrm{~K}$ is shown in Fig. $5(\mathrm{~g})$. We note that the linearity of the curve holds satisfactorily for $0.15 \leqslant B \leqslant 1.2 \mathrm{~T}$, as indicated by the arrows. The linearity 

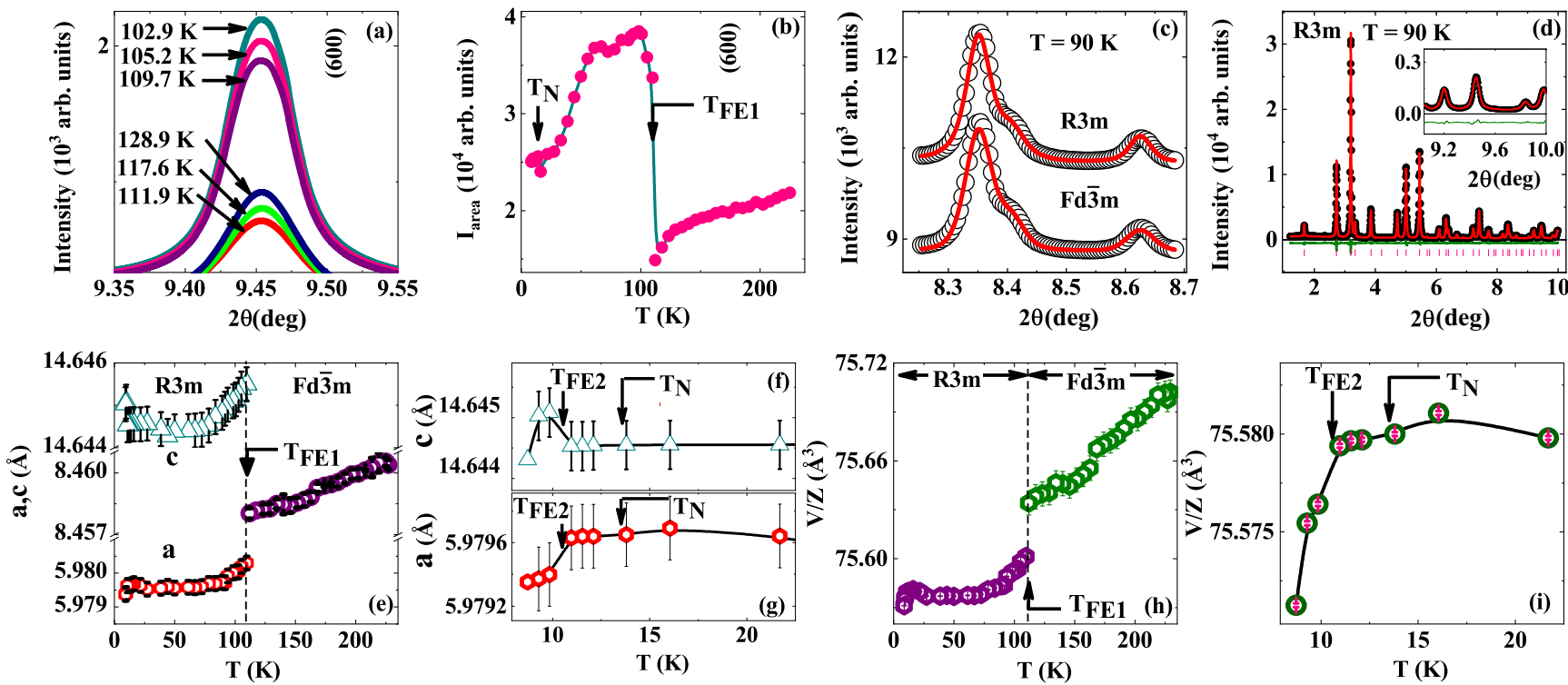

FIG. 6. (a) High- $2 \theta$ peaks shown at different $T$ around $T_{\mathrm{FE} 1}$ at $110 \mathrm{~K}$. (b) Integrated intensity ( $\left.I_{\text {area }}\right)$ of (600) peak with $T$, showing arrows at $T_{\mathrm{FE} 1}$ and $T_{N}$. Comparison of (c) the refinements (red solid curve) of the selected $2 \theta$ peaks at $90 \mathrm{~K}$ using $F d \overline{3} m$ and $R 3 m$ space groups. Rietveld refinement of the (d) diffraction pattern at $90 \mathrm{~K}$ using $R 3 \mathrm{~m}$ structure. The inset amplifies the result on the quality of refinement in a selected high- $2 \theta$ region. The $T$ variations of (e) refined lattice constants, $a$ and $c$ in range of 8.7-250 K, (f) $c$, and (g) $a$ at low- $T$ with $R 3 m$ structure, highlighting $T_{\mathrm{FE} 2}$ and $T_{N}$ by the arrows, (h) $V$ in the entire $T$ range, and (i) $V$ at low- $T$, showing $T_{\mathrm{FE} 2}$ and $T_{N}$.

of the plot indicates that the ME coupling term $\gamma P^{2} M^{2}$ of the Ginzburg-Landau theory is significant for ZFO, as reported earlier for the different multiferroics [14,45,47,61-63].

\section{G. Structural correlation to the ferroelectric order}

The structural properties are investigated using the synchrotron diffraction studies over a wide temperature range of 8-250 K and at $300 \mathrm{~K}$. The ZFO crystallizes in the normal spinel structure with the cubic $F d \overline{3} m$ (No. $227, Z=8$ ) space group at $300 \mathrm{~K}$. At $300 \mathrm{~K}$, the diffraction pattern is satisfactorily refined (not shown here) with the lattice constant, $a=$ 8.4658(3) $\AA$, considering Zn $(x, x, x)$ with $x=0.3750(0), \mathrm{Fe}$ $(0,0,0)$, and $\mathrm{O}(x, x, x)$ with $x=0.2412(9)$. The reliability parameters, $R_{w}(\%) \sim 6.60, R_{\exp }(\%) \sim 3.39$, and $\chi^{2}(\%) \sim$ 1.94, are found to be reasonable. Figure 6(a) exhibits a peak in a selected high- $2 \theta$ region, highlighting the $T$-region close to $T_{\mathrm{FE} 1}$ in the range of $102.9-128.9 \mathrm{~K}$. Here, the peaks at 109.7 and $111.9 \mathrm{~K}$ are the closest to $T_{\mathrm{FE} 1}$. From the high temperature, the intensities of the peaks decrease systematically up to $111.9 \mathrm{~K}$, below which they suddenly increase at $109.7 \mathrm{~K}$. The integrated intensity $\left(I_{\text {area }}\right)$, as estimated by the area under the (600) peak, is depicted with $T$ in Fig. 6(b). $I_{\text {area }}$ decreases systematically with decreasing $T$ up to $111.9 \mathrm{~K}$, below which a steplike sharp increase of $I_{\text {area }}$ is observed, which is followed by a further increase up to $\sim 100 \mathrm{~K}$. Below $100 \mathrm{~K}$, the $I_{\text {area }}$ decreases until $\sim 50 \mathrm{~K}$, below which it shows a steep fall down to $\sim 25 \mathrm{~K}$. A slow decrease in $I_{\text {area }}$ is observed below $\sim 25 \mathrm{~K}$.

This intensity change around $T_{\mathrm{FE} 1}$ is remarkably high as $\sim 73 \%$, which is much larger than the results observed for the multiferroics, involving the steplike isostructural changes $[13,14]$ and the structural transitions to the lower symmetries $[7,8,45,64]$. We note that the refinement is not satisfactory below $T_{\mathrm{FE} 1}$ with the $F d \overline{3} m$ structure. The examples of the refinement of the selected $2 \theta$ peaks at $90 \mathrm{~K}$ with the $F d \overline{3} m$ structure are shown in Fig. 6(c) by the continuous curves. We use the ISODISTORT [65] software to find out the possible subgroup structures at the proposed structural transition close to $T_{\mathrm{FE} 1}$. We note that $F \overline{4} 3 m$ (no. 216 ), $R \overline{3} m$ (no. 166), and $R 3 m$ (no. 160) are the possible structures with high symmetries. The refinements are performed using all the space groups, which provide the values of the reliability parameters as $R_{w}(\%) \sim 7.07,6.86,7.33$, and 5.96; $R_{\exp }(\%) \sim 3.39$, 5.04, 3.33, and 3.37; and $\chi^{2}(\%) \sim 2.08,2.07,2.20$, and 1.50, respectively, for $F d \overline{3} m, F \overline{4} 3 m, R \overline{3} m$, and $R 3 m$. Out of these structures, the $R 3 \mathrm{~m}$ space group is a polar structure and fits the best. The comparison of the refinements at $90 \mathrm{~K}$ with both the high- $T F d \overline{3} m$ and the proposed $R 3 m$ space group is shown in Fig. 6(c). A satisfactory refinement of the diffraction pattern using $R 3 \mathrm{~m}$ space group at $90 \mathrm{~K}$ is depicted by the solid curve in Fig. 6(d), where the inset shows the satisfactory refinement in a selected $2 \theta$ region. The refined coordinates are $\mathrm{Zn} 1(0,0,0.375), \mathrm{Zn} 2(0,0,0.675), \mathrm{Fe} 1(0,0,0), \mathrm{Fe} 2$ $[0.8333(4), 0.1666(7), 0.1666(7)], \mathrm{O} 1[0,0,0.2257(9)], \mathrm{O} 2$ $[0.1641(8), 0.8357(6), 0.0566(1)], \mathrm{O} 3[0,0,0.7525(3)]$, and $\mathrm{O} 4[0.502(0), 0.497(0), 0.2339(0)]$ at $90 \mathrm{~K}$.

Thermal variations of the lattice constants, as obtained from the refinement, are depicted in Fig. 6(e). With decreasing $T$, the lattice constant $(a)$, crystallized in $F d \overline{3} m$, decreases almost systematically down to $T_{\mathrm{FE} 1}$. A structural transition to a polar $R 3 m$ space group is proposed at $T_{\mathrm{FE} 1}$, as depicted in the figure. The lattice constants $c$ and $a$ close to $T_{N}$ and $T_{\mathrm{FE} 2}$ are highlighted with $T$ in Figs. 6(f) and 6(g), respectively. As shown in Fig. 6(g), the $a$ exhibits a discontinuous fall at $T_{\text {FE2 }}$. The $c(T)$ in Fig. 6(f) exhibits a broadened maximum in $a(T)$ close to $T_{\mathrm{FE} 2}$. The unit-cell volume $(V)$ is shown in Fig. $6(\mathrm{~h})$, which exhibits a steplike change at $T_{\mathrm{FE} 1}$, pointing to a first-order phase transition. We note that the contraction 
of $V$ below $T_{\mathrm{FE} 1}$ is remarkable as $\sim 0.04 \%$. The $T$-variation at low- $T$ is further highlighted in Fig. 6(i). The weak signature of $T_{N}$ may be noted in $V(T)$, pointing to a magnetoelastic coupling. However, a definite signature in $V(T)$ is observed at $T_{\mathrm{FE} 2}$, below which the unit-cell volume contracts sharply. The result proposes that further structural distortion in the polar $R 3 m$ structure correlates with the occurrence of another FE transition at low- $T$.

The FE order in $\mathrm{AFe}_{2} \mathrm{O}_{4}$-type ferrite has been reported for $\mathrm{Fe}_{3} \mathrm{O}_{4}[66,67]$ and $\mathrm{NiFe}_{2} \mathrm{O}_{4}[8]$. The origin of the $\mathrm{FE}$ order in $\mathrm{ZFO}$ is different from the recently observed ferroelectricity in $\mathrm{NiFe}_{2} \mathrm{O}_{4}$ as well as $\mathrm{Fe}_{3} \mathrm{O}_{4}$, having inverse spinel structure at room temperature [8]. Here, $T_{\mathrm{FE} 1}$ is observed at much higher than $T_{N}$, whereas the $T_{\mathrm{FE} 1}$ in $\mathrm{NiFe}_{2} \mathrm{O}_{4}$ and $\mathrm{Fe}_{3} \mathrm{O}_{4}$ was reported much below $T_{N}$ and found to correlate with the structural distortions. Here, the appearance of the FE order at $T_{\mathrm{FE} 1}=110 \mathrm{~K}$ is a rare consequence, because the FE order is associated with a short-range magnetic order, which is much above $T_{N}$ $\left(\sim 8\right.$ times $\left.T_{N}\right)$. Current results are analogous to the proposed short-range order driven occurrence of the FE order from the recent calculations [68]. In fact, the FE order coexisting with the SRO has been reported in a few occasions, such as spinel chromate [13], and a few spin-chain compounds [45,69]. Additionally, we note that the ME coupling is considerably below $T_{\mathrm{FE} 1}$. This has also been observed for the spin-chain compounds exhibiting FE order, which was associated with SRO $[45,69]$. The increase in $P$-value in ZFO may be correlated with the additional structural distortion in multiferroic $\mathrm{CoCr}_{2} \mathrm{~S}_{4}$, driven by the magnetic field [14]. We further note that the $P$-value below $T_{N}$ for ZFO does not change convincingly with the magnetic field when a separate polarization measurement is carried out close to $T_{N}$, as evident in Fig. 5(d). The result infers that neither the long-range-ordered state nor the structural distortion, giving rise to the additional electric polarization below $T_{N}$, is influenced by the magnetic field. Thus, the more plausible reason for the change in $P$-value in the high- $T$ region due to magnetic field may involve the coexistence of electric polarization with SRO, where the spins are easily polarized by the magnetic field in the SRO state. Therefore, the role of SRO needs to be explored for improving ME coupling in multiferroics.

The ZFO experiences a large magnetic frustration, as indicated by the large $\Theta / T_{N}$ ratio [26], which is attributed to the pyrochlore structure formed by the $\mathrm{Fe}$ atoms in the spinel structure. Recently, the release of geometric magnetic frustration has been proposed by a strong isostructural transition in the extensively investigated $\mathrm{CaBaCo}_{4} \mathrm{O}_{7}$ [70], which was found to be associated with the occurrence of FE order [71-74]. Here, we observe a structural transition to a polar $R 3 m$ structure from the cubic spinel structure, at which a FE order appears, coexisting with the SRO in ZFO. The result may suggest that the release of the geometric magnetic frustration due to the antiferromagnetically exchange-coupled pyrochlore structure correlates with the strong structural transition. We further note that the structural change at $T_{\mathrm{FE} 1}$ in $\mathrm{ZFO}$ is quite analogous to the most studied multiferroic $\mathrm{BiFeO}_{3}$, where the $R 3 m$ structure provides a rhombohedral distortion of the pseudocubic high- $T$ perovskite structure formed by the $\mathrm{Bi}$ atoms. This structural distortion correlates with emergence of the FE order [37,38].
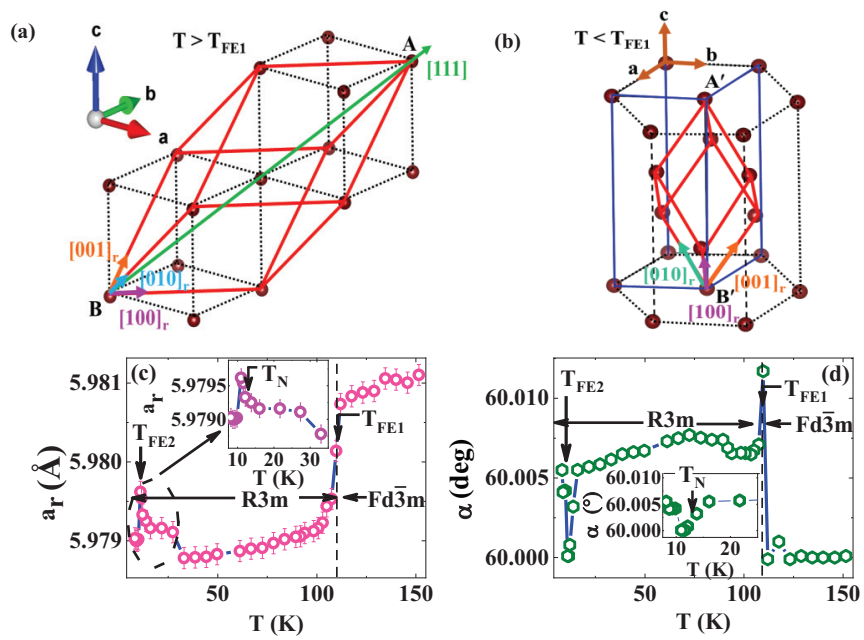

FIG. 7. (a) Connecting cubic unit cell along the [111] direction formed by $\mathrm{Fe} 1(0,0,0)$ atoms at the corner of the unit cell of $F d \overline{3} m$ structure and a rhombohedral structure shown by the thick red colored lines. Axes of the rhombohedral structure are defined as $[100]_{r},[010]_{r}$, and $[001]_{r}$. (b) Hexagonal structure formed by Fe1 $(0,0,0)$ atoms crystallized in $R 3 m$ space group and formation of a rhombohedral structure. The $T$ variations of (c) lattice constants, $a_{r}$, and (d) $\alpha$ of the isohedral rhombohedron, showing $T_{\mathrm{FE} 1}, T_{\mathrm{FE} 2}$, and $T_{N}$ by the arrows.

Here, the structural transition to the $R 3 m$ space group from the cubic spinel structure also involves the strong rhombohedral distortions, as produced by the Fe atoms in ZFO. The results of the rhombohedral distortion, for example led by the Fe1 atoms, are summarized in Fig. 7. Here, the single $\mathrm{Fe}(0,0,0)$ site in the $F d \overline{3} m$ structure divides into two $\mathrm{Fe}$ sites $(\mathrm{Fe} 1$ and $\mathrm{Fe} 2)$ in the $R 3 m$ structure. We note that the $\mathrm{Fe} 2$ atoms also produce similar distortion. Figure 7(a) depicts the relation between the cubic structures connected along the [111] direction, as indicated by the green arrow, which are formed by the $\mathrm{Fe} 1(0,0,0)$ atoms in the $F d \overline{3} m$ structure, and a pseudorhombohedral structure, as indicated by the thick red lines. These atomic arrangements are analogous to the atomic arrangements of $\mathrm{Bi}(0,0,0)$ in the cubic perovskite structure of $\mathrm{BiFeO}_{3}$ [37]. In the figure, the three arrows located at $B$ are the axes of the rhombohedral structure, which are described as $[100]_{r},[010]_{r}$, and $[001]_{r}$. Here, $A B$ is defined as $d_{[111]}$, which is the diagonal distance along the [111] direction of the rhombohedral structure. Below the structural transition, the possible rhombohedral structure formed by the Fe1 $(0,0,0)$ atoms, crystallized in the $R 3 \mathrm{~m}$ space group, is defined by the thick red lines, as depicted in Fig. 7(b). Similar to that defined in Fig. 7(a), the arrows at $B^{\prime}$ are the axes of the rhombohedral structure. In the $R 3 m$ structure, the $c$ axis direction exactly matches with the [111] direction of the isohedral rhombohedron, which is defined along the $B^{\prime} A^{\prime}$ direction in Fig. 7(b).

Thermal variations of the rhombohedral lattice constant, $a_{r}$, and the angle $(\alpha)$ of the isohedral rhombohedron are depicted in Figs. 7(c) and 7(d), respectively. The steplike decrease in $a_{r}(T)$, which is significant as $\sim 0.02 \%$, is observed close to $T_{\mathrm{FE} 1}$. Here, the $a_{r}(T)$ is considered as half of $A B$ in Fig. 7(a), having high- $T F d \overline{3} m$ structure. Below $T_{\mathrm{FE} 1}$, the $a_{r}(T)$ decreases systematically until $\sim 30 \mathrm{~K}$, below which an 
anomalous increase in $a_{r}(T)$ is observed, as highlighted in the inset of Fig. 7(c). Below $T_{N}$ it shows a sharp peak, around which $T_{\text {FE2 }}$ is observed, as indicated by an arrow in Fig. $7(\mathrm{c})$. As depicted in Fig. 7(d), $\alpha(T)$ is nearly $T$-independent above the structural transition, and a steplike rise in $\alpha$ is observed from $60^{\circ}$ to $60.011^{\circ}$. Below $T_{\mathrm{FE} 1}, \alpha(T)$ shows a broadened maximum around $\sim 70 \mathrm{~K}$, following a slow decrease. A sharp "dip" in $\alpha(T)$ is observed at $T_{\mathrm{FE} 2}$, as indicated by an arrow in Fig. 7(d), around which a sharp peak in $a_{r}(T)$ is also noted. Therefore, another rhombohedral distortion at $T_{\mathrm{FE} 2}$ correlates the low- $T$ FE order. The possible glassy magnetic state and/or AFM order might also be involved with the occurrence of low$T$ FE order. We note that the geometric magnetic frustration driven by the pyrochlore structure formed by the $\mathrm{Fe}$ atoms in $\mathrm{ZnFe}_{2} \mathrm{O}_{4}$ plays a significant role, which correlates with the glassy magnetic behavior and the occurrence of AFM order at reduced temperature. Thus, the structural distortion, glassy magnetic state, and AFM order correlate with the observed low- $T$ FE order, which needs to be explored in further studies.

\section{SUMMARY AND CONCLUSION}

In summary, the experimental observations are focused mainly on two issues: (i) the high- $T$ results close to $T_{\mathrm{FE} 1}$ and (ii) the low- $T$ results below $T_{N}$. The high- $T$ results are focused on the structural transition to a polar $R 3 m$ space group from the cubic spinel structure close to $\sim 110 \mathrm{~K}$. The strong rhombohedral distortion at the structural transition leads to the emergence of the spontaneous electric polarization, which is associated with a short-range magnetic order. A considerable ME coupling, as indicated by a $\sim 7.2 \%$ increase in polarization due to a $5 \mathrm{~T}$ magnetic field at the liquid nitrogen temperature, is noted below $T_{\mathrm{FE} 1}$. Intriguingly, the FE polarization is further increased below $T_{N}$, which links to another anomalous rhombohedral distortion.

The low- $T$ magnetization results reveal the intricate magnetic phase separation between a glassy magnetic component and an AFM component, proposing a reentrant spin-glasslike state. The magnetic memory effect proposes the glassy magnetic component below $T_{N}$. The pinning mechanism at the interface between these two magnetic components driven by the magnetic field-cooling leads to the EB effect below $T_{N}$. $\mathrm{ZnFe}_{2} \mathrm{O}_{4}$ attracts the attention of the community due to the rare occurrence of multiferroic order, which correlates closely with the strong rhombohedral distortion, analogous to that observed in $\mathrm{BiFeO}_{3}$.

\section{ACKNOWLEDGMENTS}

Portions of this work were carried out at the light source PETRA III of DESY, a member of the Helmholtz Association (HGF). Financial support (Proposal No. I-20170178) by the Department of Science \& Technology (Government of India) provided within the framework of the India@DESY collaboration is gratefully acknowledged. J.K.D. would like to acknowledge DST-INSPIRE, India fellowship.
[1] P. G. Radaelli, Y. Horibe, M. J. Gutmann, H. Ishibashi, C. H. Chen, R. M. Ibberson, Y. Koyama, Y.-S. Hor, V. Kiryukhin, and S.-W. Cheong, Nature (London) 416, 155 (2002).

[2] A. Krimmel, M. Mücksch, V. Tsurkan, M. M. Koza, H. Mutka, and A. Loidl, Phys. Rev. Lett. 94, 237402 (2005).

[3] V. Fritsch, J. Hemberger, N. Büttgen, E.-W. Scheidt, H.-A. Krug von Nidda, A. Loidl, and V. Tsurkan, Phys. Rev. Lett. 92, 116401 (2004).

[4] A. Krimmel, V. Tsurkana, D. Sheptyakov, and A. Loidl, Physica B 378-380, 583 (2006).

[5] S. Giri, H. Nakamura, and T. Kohara, Phys. Rev. B 72, 132404 (2005).

[6] P. T. Barton, M. C. Kemei, M. W. Gaultois, S. L. Moffitt, L. E. Darago, R. Seshadri, M. R. Suchomel, and B. C. Melot, Phys. Rev. B 90, 064105 (2014).

[7] A. Chatterjee, J. K. Dey, S. Majumdar, A.-C. Dippel, O. Gutowski, M. v. Zimmermann, and S. Giri, Phys. Rev. Mater. 3, 104403 (2019).

[8] J. K. Dey, A. Chatterjee, S. Majumdar, A.-C. Dippel, O. Gutowski, M. v. Zimmermann, and S. Giri, Phys. Rev. B 99, 144412 (2019).

[9] K. Tomiyasu, J. Fukunaga, and H. Suzuki, Phys. Rev. B 70, 214434 (2004).

[10] Y. Amiel, G. Kh. Rozenberg, N. Nissim, A. Milner, M. P. Pasternak, M. Hanfland, and R. D. Taylor, Phys. Rev. B 84, 224114 (2011).

[11] Y. Yamasaki, S. Miyasaka, Y. Kaneko, J.-P. He, T. Arima, and Y. Tokura, Phys. Rev. Lett. 96, 207204 (2006).
[12] Q. Zhang, K. Singh, F. Guillou, C. Simon, Y. Breard, V. Caignaert, and V. Hardy, Phys. Rev. B 85, 054405 (2012).

[13] K. Dey, S. Majumdar, and S. Giri, Phys. Rev. B 90, 184424 (2014).

[14] K. Dey, A. Karmakar, A. Indra, S. Majumdar, U. Rütt, O. Gutowski, M. v. Zimmermann, and S. Giri, Phys. Rev. B 92, 024401 (2015).

[15] S.-H. Lee, H. Takagi, D. Louca, M. Matsuda, S. Ji, H. Ueda, Y. Ueda, T. Katsufuji, J.-H. Chung, S. Park, S.-W. Cheong, and C. Broholm, J. Phys. Soc. Jpn. 79, 011004 (2010).

[16] A. P. Ramirez, Annu. Rev. Mater. Sci. 24, 453 (1994).

[17] J. S. Gardner, M. J. P. Gingras, and J. E. Greedan, Rev. Mod. Phys. 82, 53 (2010).

[18] S. T. Bramwell and M. J. P. Gingras, Science 294, 1495 (2001).

[19] S. T. Bramwell, M. J. Harris, B. C. den Hertog, M. J. P. Gingras, J. S. Gardner, D. F. McMorrow, A. R. Wildes, A. Cornelius, J. D. M. Champion, R. G. Melko, and T. Fennell, Phys. Rev. Lett. 87, 047205 (2001).

[20] K. Matsuno, T. Katsufuji, S. Mori, Y. Moritomo, A. Machida, E. Nishibori, M. Takata, M. Sakata, N. Yamamoto, and H. Takagi, J. Phys. Soc. Jpn. 70, 1456 (2001).

[21] K. Matsuno, T. Katsufuji, S. Mori, M. Nohara, A. Machida, Y. Moritomo, K. Kato, E. Nishibori, M. Takata, M. Sakata, K. Kitazawa, and H. Takagi, Phys. Rev. Lett. 90, 096404 (2003).

[22] G. B. Sim and S. B. Lee, Phys. Rev. B 98, 014423 (2018).

[23] Y. V. Tymoshenko, Y. A. Onykiienko, T. Múller, R. Thomale, S. Rachel, A. S. Cameron, P. Y. Portnichenko, D. V. Efremov, V. Tsurkan, D. L. Abernathy, J. Ollivier, A. Schneidewind, A. 
Piovano, V. Felea, A. Loidl, and D. S. Inosov, Phys. Rev. X 7, 041049 (2017).

[24] J. M. Hastings and L. M. Corliss, Rev. Mod. Phys. 25, 114 (1953).

[25] W. Schiessl, W. Potzel, H. Karzel, M. Steiner, G. M. Kalvius, A. Martin, M. K. Krause, I. Halevy, J. Gal, W. Schäfer, G. Will, M. Hillberg, and R. Wäppling, Phys. Rev. B 53, 9143 (1996).

[26] K. Kamazawa, Y. Tsunoda, H. Kadowaki, and K. Kohn, Phys. Rev. B 68, 024412 (2003).

[27] D. J. Singh, M. Gupta, and R. Gupta, Phys. Rev. B 63, 205102 (2001).

[28] C. Cheng, Phys. Rev. B 78, 132403 (2008).

[29] M. Gaudon, N. Pailhé, A. Wattiaux, and A. Demourgues, Mater. Res. Bull. 44, 479 (2009).

[30] J. Melo Quintero, K. L. Salcedo Rodríguez, G. A. Pasquevich, P. Mendoza Zélis, S. J. Stewart, C. E. Rodríguez Torres, and L. A. Errico, Hyperfine Interact. 237, 63 (2016).

[31] E. Greenberg, W. M. Xu, M. Nikolaevsky, E. Bykova, G. Garbarino, K. Glazyrin, D. G. Merkel, L. Dubrovinsky, M. P. Pasternak, and G. Kh. Rozenberg, Phys. Rev. B 95, 195150 (2017).

[32] K. L. Salcedo Rodríguez, S. J. Stewart, P. M. Mendoza Zélis, G. A. Pasquevich, and C. E. Rodríguez Torres, J. Alloys Compd. 752, 289 (2018).

[33] J. J. Melo Quintero, C. E. Rodríguez Torres, and L. A. Errico, J. Alloys Compd. 741, 746e755 (2018).

[34] K. Kamazawa, Y. Tsunoda, K. Odaka, and K. Kohn, J. Phys. Chem. Solids 60, 1261 (1999).

[35] K. Kamazawa, S. Katano, and Y. Tsunoda, Physica B 345, 96 (2004).

[36] Y. Fei, S. J. Kennedy, S. J. Campbell, and M. Hofmann, Physica B 356, 264 (2005).

[37] F. Kubel and H. Schmid, Acta Crystallogr. B46, 698 (1990).

[38] J.-G. Park, M. D. Le, J. Jeong, and S. Lee, J. Phys.: Condens. Matter 26, 433202 (2014).

[39] Y. Hou, X. Li, Q. Zhaoa, and G. Chen, Appl. Catal. B 142, 80 (2013).

[40] M. Wang, Z. Ai, and L. Zhang, J. Phys. Chem. C 112, 13163 (2008).

[41] H. Song, L. Zhu, Y. Li, Z. Lou, M. Xiao, and Z. Ye, J. Mater. Chem. A 3, 8353 (2015).

[42] M. I. Mendelson, J. Am. Ceram. Soc. 52, 443 (1969).

[43] J. A. Mydosh, Spin Glasses: An Experimental Introduction (Taylor \& Francis, London, 1993).

[44] Y. Sun, M. B. Salamon, K. Garnier, and R. S. Averback, Phys. Rev. Lett. 91, 167206 (2003).

[45] A. Indra, K. Dey, S. Majumdar, I. Sarkar, S. Francoual, R. P. Giri, N. Khan, P. Mandal, and S. Giri, Phys. Rev. B 95, 094402 (2017).

[46] D. De, A. Karmakar, M. K. Bhunia, A. Bhaumik, S. Majumdar, and S. Giri, J. Appl. Phys. 111, 033919 (2012).

[47] J. K. Dey, S. Majumdar, and S. Giri, J. Phys.: Condens. Matter 30, 235801 (2018).

[48] S. Giri, M. Patra, and S. Majumdar, J. Phys.: Condens. Mater. 23, 073201 (2011).

[49] W. H. Meiklejohn and C. P. Bean, Phys. Rev. 102, 1413 (1956).
[50] W. H. Meiklejohn and C. P. Bean, Phys. Rev. 105, 904 (1957).

[51] J. Nogues and I. K. Schuller, J. Magn. Magn. Mater. 192, 203e232 (1999).

[52] P. J. van der Zaag, A. R. Ball, L. F. Feiner, and R. M. Wolf, J. Appl. Phys. 79, 5103 (1996).

[53] P. J. van der Zaag, R. M. Wolf, A. R. Ball, C. Bordel, L. F. Feiner, and R. Jungblut, J. Magn. Magn. Mater. 148, 346 (1995).

[54] S. Sabyasachi, M. Patra, S. Majumdar, S. Giri, S. Das, V. S. Amaral, O. Iglesias, W. Borghols, and T. Chatterji, Phys. Rev. B 86, 104416 (2012).

[55] M. Thakur, M. Patra, K. De, S. Majumdar, and S. Giri, J. Phys.: Condens. Matter 20, 195215 (2008).

[56] K. De, M. Patra, S. Majumdar, and S. Giri, J. Phys. D 41, 175007 (2008).

[57] P. M. Shand, T. Rash, M. Streicher, T. E. Kidd, K. R. Boyle, and L. H. Strauss, Phys. Rev. B 82, 214413 (2010).

[58] T. N. M. Ngo, U. Adem, and T. T. M. Palstra, Appl. Phys. Lett. 106, 152904 (2015).

[59] N. Terada, Y. S. Glazkova, and A. A. Belik, Phys. Rev. B 93 155127 (2016).

[60] R. Chen and Y. Kirsh, Analysis of Thermally Stimulated Process (Pergamon, New York, 1981).

[61] T. D. Sparks, M. C. Kemei, P. T. Barton, R. Seshadri, E.-D. Mun, and V. S. Zapf, Phys. Rev. B 89, 024405 (2014).

[62] N. Mufti, A. A. Nugroho, G. R. Blake, and T. T. M. Palstra, J. Phys.: Condens. Matter 22, 075902 (2010).

[63] T. Kimura, S. Kawamoto, I. Yamada, M. Azuma, M. Takano, and Y. Tokura, Phys. Rev. B 67, 180401(R) (2003).

[64] A. Indra, K. Dey, J. K. Dey, S. Majumdar, U. Rütt, O. Gutowski, M. v. Zimmermann, and S. Giri, Phys. Rev. B 98, 014408 (2018).

[65] B. J. Campbell, H. T. Stokes, D. E. Tanner, and D. M. Hatch, J. Appl. Crystallogr. 39, 607 (2006).

[66] K. Kato and S. Iida, J. Phys. Soc. Jpn. 51, 1335 (1982).

[67] Y. Miyamoto, M. Kobayashi, and S. Chikazumi, J. Phys. Soc. Jpn. 55, 660 (1986).

[68] X. Wan, H.-C. Ding, S. Y. Savrasov, and C.-G. Duan, Sci. Rep. 6, 22743 (2016).

[69] T. Basu, V. V. Ravi Kishore, S. Gohil, K. Singh, N. Mohapatra, S. Bhattacharjee, B. Gonde, N. P. Lalla, P. Mahadevan, S. Ghosh, and E. V. Sampathkumaran, Sci. Rep. 4, 5636 (2014).

[70] V. Caignaert, V. Pralong, V. Hardy, C. Ritter, and B. Raveau, Phys. Rev. B 81, 094417 (2010).

[71] V. Caignaert, A. Maignan, K. Singh, Ch. Simon, V. Pralong, B. Raveau, J. F. Mitchell, H. Zheng, A. Huq, and L. C. Chapon, Phys. Rev. B 88, 174403 (2013).

[72] R. S. Fishman, S. Bordács, V. Kocsis, I. Kézsmárki, J. Viirok, U. Nagel, T. Röm, A. Puri, U. Zeitler, Y. Tokunaga, Y. Taguchi, and Y. Tokura, Phys. Rev. B 95, 024423 (2017).

[73] K. Dey, A. Indra, A. Chatterjee, S. Majumdar, U. Rütt, O. Gutowski, M. v. Zimmermann, and S. Giri, Phys. Rev. B 96, 184428 (2017).

[74] C. Dhanasekhar, A. K. Das, Ripandeep Singh, A. Das, G. Giovannetti, D. Khomskii, and A. Venimadhav, Phys. Rev. B 96, 134413 (2017). 